\title{
Central retinal vein occlusion-an underappreciated association with obstructive sleep apnoea syndrome
}

\author{
ZX Yeoh*, M Ayzam Ahmad, M Masaany and Siti Sabzah MH \\ Department of Otorhinolaryngology, Hospital Sultanah Bahiyah, Alor Setar, Kedah, Malaysia
}

\begin{abstract}
Obstructive Sleep Apnoea Syndrome (OSAS) is a sleep disorder with ceasation or significant decrease in airflow in the presence of breathing effort. As episodic oxyhaemoglobin desaturation occurred throughout sleep, it causes sleep fragmentation, increasing breathing work, alveolar hypoventilation and intermittent hypoxaemia. Several ophthalmologic conditions have been found to have a clear association with OSAS and indeed may be the presenting symptoms of OSAS, but unfortunately the awareness about it is still low among medical professionals. We present a case report of a young and otherwise healthy gentleman who developed sudden-onset left eye blurred vision without any preceding trauma or associated acute raised intracranial pressure symptoms. Opthalmologic assessment concluded an episode of left acute central retinal vein occlusion (RVO). Assessment for metabolic disease showed normal investigation parameters. Further history revealed nocturnal snoring with daytime somnolence for years. Subsequent work-up confirmed moderate OSAS. After utilizing home nocturnal Continuous Positive Airway Pressure (CPAP) machine for 2 months, his OSAS symptoms significantly improved, in tandem with his vision and ophthalmologic findings. While it is scientifically early to conclude OSAS treatment can modify course of RVO or prevent recurrence on second eye, it is vital for physicians to be aware of its association as OSAS treatment has demonstrated efficacy in reducing other more well-established risk of cardiovascular and cerebrovascular disease.
\end{abstract}

\section{Introduction}

Obstructive Sleep Apnoea Syndrome (OSAS) is a sleep-related breathing disorder that involves ceasation or significant decrease in airflow despite ongoing effort to breathe [1]. Muscle relaxation during sleep causes soft tissue collapse in upper airway thus leading to partial reductions (hypopnoea) and complete pauses (apnoea) in breathing. As episodic oxyhaemoglobin desaturation occurred throughout sleep, it causes sleep fragmentation, increasing breathing work, alveolar hypoventilation and intermittent hypoxaemia [2]. OSAS is associated with several ophthalmologic conditions which may not be as readilyknown even among medical practitioners. We present a case report of an opthalmologic condition seemingly-caused by OSAS which was even undiagnosed before then.

\section{Case report}

A 37 years old gentleman was referred to Ophthalmology Clinic for sudden-onset left eye blurred vision for 1 week prior to presentation. He described a sensation of his vision was "blocked by curtain" with associated eye discomfort. He had minimal floaters which was not increasing in size and was wearing glasses for past 18 years for high myopia (near-sightedness) but otherwise he had no history of eye trauma, head injury or associated acute raised intracranial pressure symptoms. He had no other significant medical comorbidities except for mild dyslipidaemia. Ophthalmologic assessment including fundoscopy (Figure 1) concluded an episode of left acute central retinal vein occlusion (CRVO) with intraocular pressure was within normal range for both eyes. Further assessments for metabolic disease including laboratory blood tests were normal.

Subsequent history-taking revealed nocturnal snoring, frequent choking with midnight awakening and daytime somnolence for many preceding years. The patient was overweight with Body Mass Index
(BMI) of 27. Intraoral examination showed grade 2 tonsillar hypertrophy with Friedman palate position III. Flexible nasopharyngoaryngoscopy demostrated prominent tongue base follicles and positive Muller's manouvre with $100 \%$ and $50 \%$ airway collapse at retropalatal and retrolingual level, respectively. Urgent full attended polysomnography confirmed severe OSAS with Apnoea-Hypopnoea Index (AHI) of 30.9

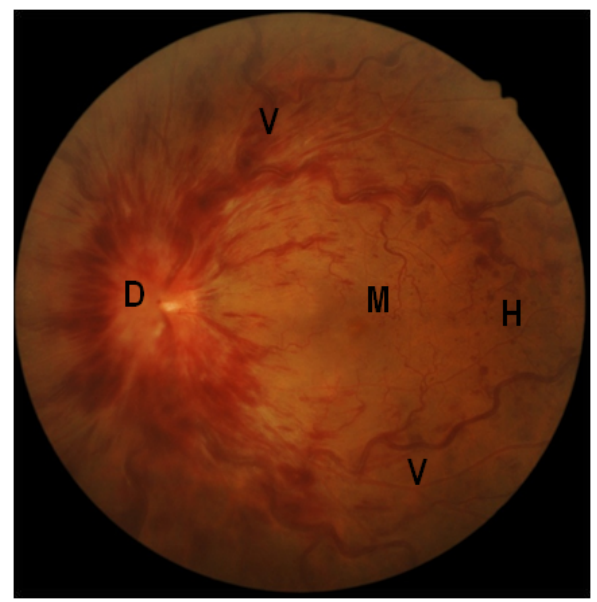

Figure 1. Left eye fundoscopy before intervention, showing swollen and elevated optic disc (D) with blurred disc margin, extensive flame-shaped haemorrhages (H), tortuous dilated vessels (V), and edematous macula (M).

Correspondence to: Dr. Yeoh Zhi Xiang, Department of Otorhinolaryngology, Hospital Sultanah Bahiyah, KM6, Jalan Langgar, 05460 Alor Setar, Kedah, Malaysia; Tel: +604-7407846; E-mail: zhixiang@hotmail.com

Key words: sleep apnoea syndrome, breathing disorder, fundoscopy

Received: June 12, 2016; Accepted: July 12, 2016; Published: July 16, 2016 


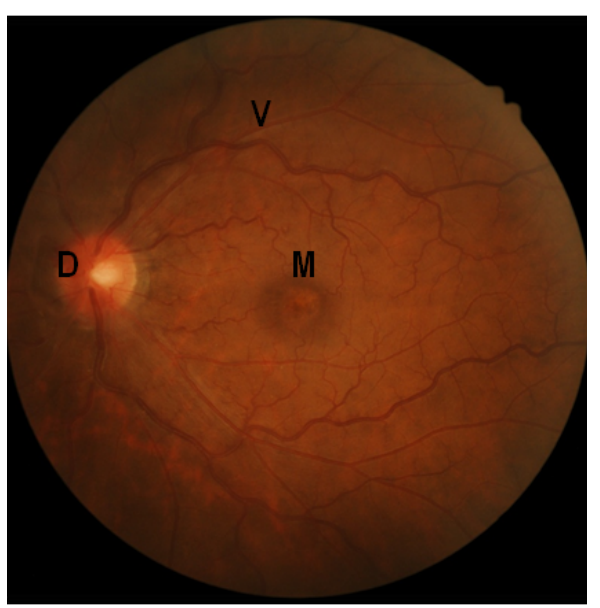

Figure 2. Left eye fundoscopy after intervention, 2 months after initial fundoscopy, showing significantly-improved findings; note the optic disc (D) margin is sharp, macula (M) is less edematous, and vessels $(\mathbf{V})$ less dilated or tortuous.

and Respiratory Disturbance Index (RDI) of 31.2.

After discussion, he opted for home Continuous Positive Airway Pressure (CPAP) therapy for 2 months, after which his OSAS symptoms improved significantly in tandem with his vision and ophthalmologic findings (Figure 2). His latest AHI dropped to 1.5 with mean autoadjusted Positive End-Expiratory Pressure (PEEP) of $6.1 \mathrm{~cm} \mathrm{H}_{2} \mathrm{O}$. In view of clinical improvement, he requested to postpone the plan for surgery. He remained well to date.

\section{Discussion}

OSAS is characterised by recurrent episodes of upper airway collapse during sleep which are associated with recurrent oxygen desaturation and arousals from sleep [2]. The nocturnal and daytime symptoms may be insidious and not known to the patient until being referred for evaluation after complications arised many years later.

Several ophthalmologic conditions have been found to have a clear association with OSAS, and may be the presenting symptoms of OSAS. These range from common but benign entity of Floppy Eyelid Syndrome, to the more vision-threatening conditions such as normotensive glaucoma and papilloedema [1]. Prevalence of OSAS is quoted as high as $27 \%$ among patients with glaucoma; OSAS patients are 4 times more likely than age-matched controls to have glaucoma or visual field defects [1]. The author thus proposed screening of glaucoma among OSAS patients, and vice versa especially if normal tension glaucoma is diagnosed in otherwise low risk profile patients [1].

Central retinal vein occlusion (CRVO) is a relatively common retinal vascular disorder with characteristic fundoscopic findings of retinal haemorrhages, dilated tortuous retinal veins, cottonwool spots, macular edema and optic disc edema [1]. Due to unique anatomical arrangement of central retina artery and vein sharing a common adventitial sheath, central retinal vein is predisposed to thrombus formation as it passes through the narrow opening of lamina cribosa. Common causes for CRVO are related to vascular wall (e.g. atherosclerosis related to diabetes mellitus, hypertension, vasculitis, etc.) and blood hypercoagulable state (e.g. polycythaemia vera, clotting disorders, etc.). There is no known definitive medical treatment for prevention or cure, and the management centres in identifying and treating underlying cause(s).

Sleep apnoeas such as OSAS has been linked with CRVO in many published papers. Chou et al. [3] in his Taiwanese population study found convincing figures of sleep apnoea being an independent risk factor for RVO with almost two times odds ratio of RVO among OSAS patients compared to control population. Crouch et al. [4] hypothesised sympathetic overactivity, which is central in much of OSAS-related complications, leads to vasoconstriction and kinking of retinal vessels manifesting as tortuousity. Loss of pericytes in diabetic patients can be an important cause but it is worth noting that our patient was normoglycaemic.

More convincing association of OSAS with RVO was welldemonstrated by Glacet-Bernard et al in his series of 63 consecutive RVO patients [5]. The author performed polysomnography (considered as gold standard in diagnosing OSAS) for RVO patients with significant OSAS symptoms, and found that the lowest prevalence rate of OSAS among RVO patients was 37\%, way higher than matching age population cohort OSAS prevalence of $11 \%$. Although a causal relationship cannot be concluded, a double vicious cycle pathophysiology has been proposed to show that OSAS, by acting on retinal microcirculation, could at the very least be an additional risk factor for occurrence of RVO. While OSA treatment may not necessarily be the treatment for CRVO in these patients, it is vital that treating physicians be aware of the association, as OSA treatment has demonstrated its efficacy in reducing risk of cardiovascular and cerebrovascular disease in affected group.

\section{Conclusion}

OSAS is often under recognized due to its varied and subtle symptoms. There is more than just a "chance" relationship between OSAS and certain ophthalmologic conditions. Although its prevalence among OSAS patients is small, it is significantly higher compared to general population. Patients presenting with ocular symptoms such as the case we outlined above should be queried regarding symptoms suggestive of OSAS and referred for further evaluation. This case highlights the importance of treating medical practitioners to be aware of its association because high index of clinical suspicion is required to diagnose OSAS and prevent further complications.

\section{References}

1. CJ Lettieri (2013) The 5 most common ocular manifestations of obstructive sleep apnea. Medscape.

2. R Downey III, Rowley JA, Wickramasinghe H, Gold PM, et al. (2014) Obstructive sleep apnoea. Medscape

3. Chou KT, Huang CC, Tsai DC, Chen YM, Perng DW, Shiao GM, et al. (2012) Sleep apnea and risk of retinal vein occlusion: a nationwide population-based study of Taiwanese. Am J Ophtalmol 154: 200-205. [Crossref]

4. ER Crouch, Thompson JE, Vorona R, Ware C, Lattanzio F Jr, Sammuel P (2005) Retinal vascular changes in obstructive sleep apnea syndrome. Invest Opthalmol Vis Sci 46: E-Abstract 3278.

5. Glacet-Bernard A, Leroux les Jardins G, Lasry S, Coscas G, Soubrane G, et al. (2010) Obstructive sleep apnea among patients with retinal vein occulusion. Arch Opthalmol 128: 1533-1538. [Crossref]

Copyright: (C2016 Yeoh ZX. This is an open-access article distributed under the terms of the Creative Commons Attribution License, which permits unrestricted use, distribution, and reproduction in any medium, provided the original author and source are credited. 